\title{
Separating inner and outer contributions in gravitational lenses with the perturbative method
}

\begin{abstract}
C. Alard
Institut d'Astrophysique de Paris, 98bis boulevard Arago, 75014 Paris, France

e-mail: alard@iap.fr

Received 2 October 2009 / Accepted 28 December 2009

ABSTRACT

Context. This paper presents a reconstruction of the gravitational lens SL2S02176-0513 with the singular perturbative method presented in Alard (2007, MNRAS, 382, L58) and Alard (2008, MNRAS, 388, 375).

Aims. The ability of the perturbative method to separate the inner and outer contributions of the potential in gravitational lenses is tested using SL2S02176-0513. The gravitational field of the central galaxy is dominated by a nearby group of galaxies located at a distance of a few critical radius in this lens.

Methods. The perturbative functionals are re-constructed using local polynomials. The polynomial interpolation is smoothed by Fourier series, and numerically fitted to the Hubble Space Telescope data with a non linear minimization procedure. The potential inside and outside the critical circle is derived from the reconstruction of the perturbative fields.

Results. The inner and outer potential contours are very different. The inner contours are consistent with the central galaxy, while the outer contours are fully consistent with the perturbation introduced by the group of galaxies.

Conclusions. The ability of the perturbative method to separate the inner and outer contribution is confirmed, and indicates that in the perturbative approach the field of the central deflector can be separated from outer perturbations. The separation of the inner and outer contribution is especially important to study the shape of dark matter halos and for the statistical analysis of the effect of dark matter substructures.
\end{abstract}

Key words. gravitational lensing: strong - dark matter - methods: data analysis

\section{Introduction}

Gravitational lenses are valuable astrophysical tools to probe the mass distribution of dark halos. The recent results obtained by Clowe et al. (2006) are a good example of the ability of gravitational lensing to probe complex mass distributions. In this context the recently perturbative approach (Alard 2007), and its application for the reconstruction of strong gravitational lenses (Alard 2008, 2009), is of particular interest since this approach relates directly to the properties of the images to the shape of the lens potential. The lens SL2S02176-0513 is the second lens to be reconstructed by the perturbative approach. The first re-construction of a gravitational lens by the perturbative approach is described in Alard (2009), this work offers an overview presentation of the methods and algorithms that will be used in this paper. The lens SL2S02176-0513 was already modeled by $\mathrm{Tu}$ et al. (2009) with conventional methods. Tu et al. (2009) identified three components in the lens: the stellar component, the dark matter halo associated with the galaxy, and an external influence from a nearby group of galaxies. The surface density of the bright part is modeled as a Sersic profile, while the dark component is represented with an elliptical pseudo isothermal sphere. The center of the dark component is supposed to be the same as the center of light. The external component is represented with a singular isothermal sphere (SIS). The position of the SIS is not a free parameter, it is derived from X-ray observations (Geach et al. 2007). The model proposed by Tu et al. (2009) is physically motivated, although it has a considerable number of free parameters, and the exploration of the parameter space is always a difficult task. The minimization surface in the parameter space is complex, with the usual presence of several minima of similar depth resulting in a degeneracy of the solution. The presence of several minima of similar depth is due to a degeneracy in the representation of the lens. This degeneracy arises because the properties of the lens are fixed once a given set of perturbative functions is adopted with the perturbative approach. However, a single set of perturbative functions does not correspond to a single model, but to a family of models which is consistent with the local constraints. In general both the halo profile steepness and its geometry are degenerate. An extreme example of this degeneracy is that observations of arcs in clusters of galaxies can be reproduced with good agreement either with a circularly symmetrical halo profile with a flat density core, or a steep density profile with a small ellipticity (Bartelmann \& Meneghetti 2004). This degeneracy corresponds to the "shape degeneracy" presented by Saha \& Williams (2006) and Zhao \& Qin (2003). Thus the problem in itself is not to find a possible solution but to explore the range of solutions consistent with the data. The family of solutions have common properties and these common properties are the really interesting quantities. The perturbative approach has the advantage to describe the lens with a general singular perturbative expansion. A given perturbative expansion corresponds to a family of models, and not to a single model. An important asset in this approach is that the general properties of the solution are directly related to the physical properties of the images. This direct relation indicates that the perturbative reconstruction is not degenerated. A general description of the perturbative methods and of their application to the inversion of gravitational lenses is available in Alard (2009). 


\section{The perturbative approach in gravitational lensing}

This section presents a brief summary of the singular perturbative approach in gravitational lensing developed in Alard (2007). The perturbation is singular since the unperturbed solution is a circle with an infinite number of points, while the perturbed solution has only a finite number of points. It is important to note that this perturbative approach is not related to the conventional regular perturbative method already explored in gravitational lensing. For an example of the regular perturbative theory in gravitational lensing, the interested reader is referred to Vegetti \& Koopmans (2009). The unperturbed situation is represented by a circular lens with a potential $\phi_{0}(r)$ and a point source with a null impact parameter. In the perturbed situation the source has an impact parameter $\boldsymbol{r}_{S}$ and the lens is perturbed by the non circular potential $\psi(r, \theta)$. Both perturbations, $\boldsymbol{r}_{S}$ and $\psi$ are assumed to be of the same order $\epsilon$

$$
\begin{aligned}
r_{S} & =\epsilon \boldsymbol{r}_{s} \\
\phi & =\phi_{0}+\epsilon \psi .
\end{aligned}
$$

Similar ideas were explored by Blandford \& Kovner (1988); the main difference with Alard (2007) is the derivation of analytical equations describing image formation. These equations directly relate the images properites to the local potential; the derivation of these equations will be presented below. Note that for convenience the unit of the coordinate system is the critical radius. Thus the critical radius by in this coordinate system is situated at $r=1$. As a consequence, in this work all distances and their associated quantities (errors,..., will be expressed in unit of the critical radius. In response to this perturbation the radial position of the image will be shifted by an amount $\mathrm{d} r$ with respect to the un-perturbed point on the critical circle. The new radial position of the image is:

$r=1+\epsilon \mathrm{d} r$.

The response $\mathrm{d} r$ to the perturbation can be evaluated by solving the lens equation in the perturbative regime, leading to the perturbative lens equation:

$\boldsymbol{r}_{\boldsymbol{s}}=\left(\kappa_{2} \mathrm{~d} r-f_{1}\right) \boldsymbol{u}_{\boldsymbol{r}}-\frac{\mathrm{d} f_{0}}{\mathrm{~d} \theta} \boldsymbol{u}_{\theta}$

and $\kappa_{2}=1-\left[\frac{\mathrm{d}^{2} \phi_{0}}{\mathrm{~d} r^{2}}\right]_{(r=1)}$

This equation corresponds to Eq. (8) in Alard (2007). Considering that the source has a mean impact parameter $\boldsymbol{r}_{\mathbf{0}}$, the position in the source plane may be rewritten as $\boldsymbol{r}_{\boldsymbol{S}}=\tilde{\boldsymbol{r}_{\boldsymbol{S}}}+\boldsymbol{r}_{\mathbf{0}}$. Assuming Cartesian coordinates $\left(x_{0}, y_{0}\right)$ for the vector $\boldsymbol{r}_{\mathbf{0}}$ Eq. (3) reads

$\tilde{\boldsymbol{r}}_{\boldsymbol{s}}=\left(\kappa_{2} \mathrm{~d} r-\tilde{f}_{1}\right) \boldsymbol{u}_{\boldsymbol{r}}-\frac{\mathrm{d} \tilde{f}_{0}}{\mathrm{~d} \theta} \boldsymbol{u}_{\boldsymbol{\theta}}$

For a circular source with a radius $R_{0}$, the perturbative response $d r$ takes the simple form (Alard 2007, Eq. (12))

$\mathrm{d} r=\frac{1}{\kappa_{2}}\left[\tilde{f}_{1} \pm \sqrt{R_{0}^{2}-\left(\frac{\mathrm{d} \tilde{f}_{0}}{\mathrm{~d} \theta}\right)^{2}}\right]$.

To conclude it is important to note that Eq. (3) depends on $\kappa_{2}$. However, this variable can be eliminated from Eq. (3) by renormalizing the fields $f_{n}=\frac{f_{n}}{\kappa_{2}}$, and the source plane coordinates, $\boldsymbol{r}_{s}=\frac{\boldsymbol{r}_{s}}{\kappa_{2}}$ (mass sheet degeneracy). These renormalized variables will be adopted throughout this work. The re-normalization is equivalent to solving Eqs. (3) and (5) for $\kappa_{2}=1$. The variable $\kappa_{2}$ will re-appear when the re-normalized quantities are converted to the original quantities.

\section{Preprocessing of the data}

The gravitational lens SL2S02176-0513 (Cabanac et al. 2007, SL2S public domain) was observed by the Hubble Space Telescope in three spectral domains, F475W, F606W, F814W with an exposure time of $400 \mathrm{~s}$. Cosmics cleaning and image reinterpolation to a common grid are performed with a software from the ISIS package (Alard 2000). Considering that in the radial direction the arc size is only a few pixels, it is useful to work on a finer grid to facilitate the numerical calculations (convolutions with the point spread function for instance). The images were remapped to a grid with a pixel size smaller by a factor of two, which is small enough to avoid aliasing problems. But note that this resampling is only a numerical convenience, and that the computing of statistical estimates is performed with the original data, and not the re-sampled and interpolated (correlated) data. Note also that at this level no astrometric registration is performed and that the coordinate system is the coordinate system of the initial HST image. Still the final result will be presented in a proper astrometric system (North up, and East left). Note also that there is a large number of cosmic rays in these images, and the local density of those is sometime very high. When too many nearby cosmics are detected within a small area, the area is flagged and will be considered cautiously, or even rejected. Finally, the three cleaned and recentered images are stacked and the background is subtracted to produce a reference image of the arc system. Two color illustrations of the arc system are also provided in Fig. 1.

\section{Lens inversion}

The lens SL2S02176-0513 forms a system of images which resembles a cusp configuration, with a large arc on the left side of the deflector and a small counter image on the other side (see Fig. 1). The 4 bright parts in the images have similar brightness and are probably the image of a unique area in the source (see Fig. 1). Tu et al. (2009) have associated another fainter bright detail with these features (see Fig. 1). But we will see that this association is unlikely as this detail is of a slightly different color and would additionally require a complicated field shape for it to be associated with the other four bright features.

\subsection{Previous work}

Note also that Tu et al. (2009) found that another system of images is associated with this lens. These images correspond to a more distant source, and the light from this second source is influenced by the potential of the first source. This lens is complex, but the associated images are small and noisy and provide only a few local constraints. Thus since there are many free parameters in this new system (mass of the first deflector, source position and shape) and few data to constrain these parameters, not much additional information on the second lens can be obtained by including this system of images in the analysis. 


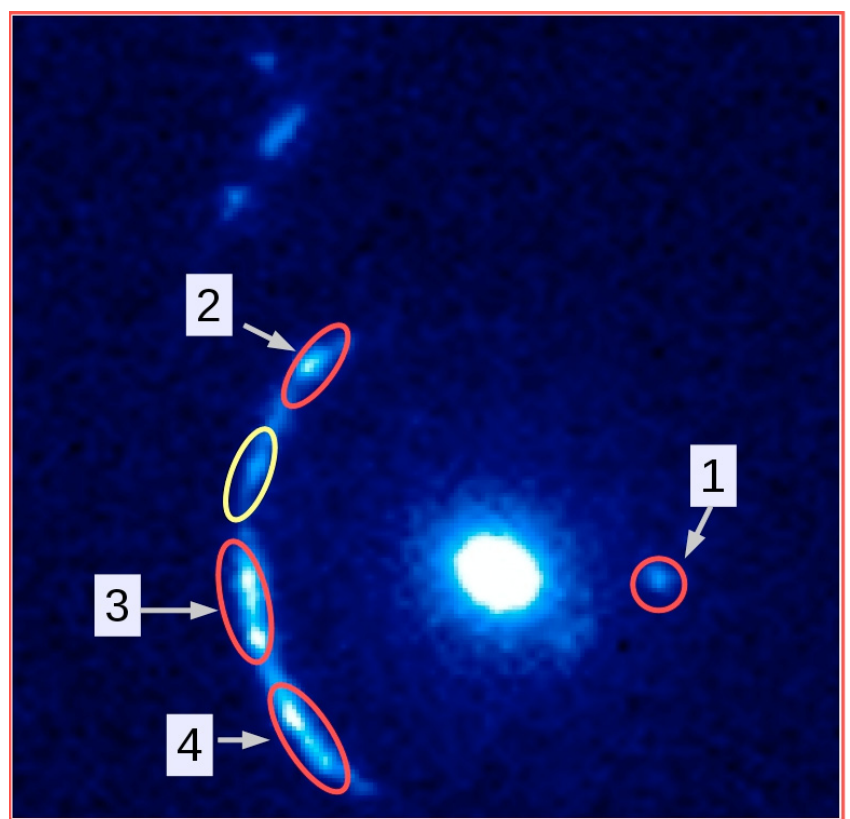

Fig. 1. The red contours indicate the four bright areas visible in the image of the source formed by the lens. The flux in these areas is similar, which suggests that these area corresponds to the same part of the source plane. The yellow contour indicates a detail associated with the 4 other bright details by Tu et al. (2009).

\subsection{Estimation of the critical circle}

The critical circle is estimated by fitting a circle to the mean position of the images. The center and radius of the circle are adjusted. The nonlinear adjustment procedure starts from a circle centered on the small galaxy at the center of the image. The initial estimate for the circle radius is the mean distance of the images to the center. A nonlinear optimization shows that the best center is close to the initial guess and that the optimal radius is close to 30.5 pixels; with a pixel size of $0.049^{\prime \prime}$ the critical circle radius $R_{\mathrm{C}}$ is: $1.49^{\prime \prime}$. Note that the final result will not depend upon the particular choice of a given critical circle. Taking another circle close to this one would change a little the estimation of the perturbative fields, but the total background plus the perturbation would remain the same.

\subsection{General properties of the solution}

An approximate solution will be derived from the properties of the circular source solution. To estimate the circular source solution, the outer contour of the bright area in the source will be approximated with a circle. Note that since the bright areas of the image are composed of two nearby bright spots, the corresponding source region is also composed of two bright features. In the circular approximation, we will consider the circular envelope of these two bright spots. It would have been possible to consider the bright spots one by one, but the determination of the local parameters would then have been less accurate. This approximation of the source may not be very accurate as the typical error on the field estimation will be on the order of the source outer contour deviations from circularity. However, this approximation is sufficient to derive the general properties of the solution, and in particular the behavior of the $\frac{\mathrm{d} f_{0}}{\mathrm{~d} \theta}$ field near the minimum. For circular sources the perturbative fields are directly related to the data (see Sect. 3, and Eq. (5)). The field $f_{1}$ corresponds to the mean radial position of the image, while the reconstruction of

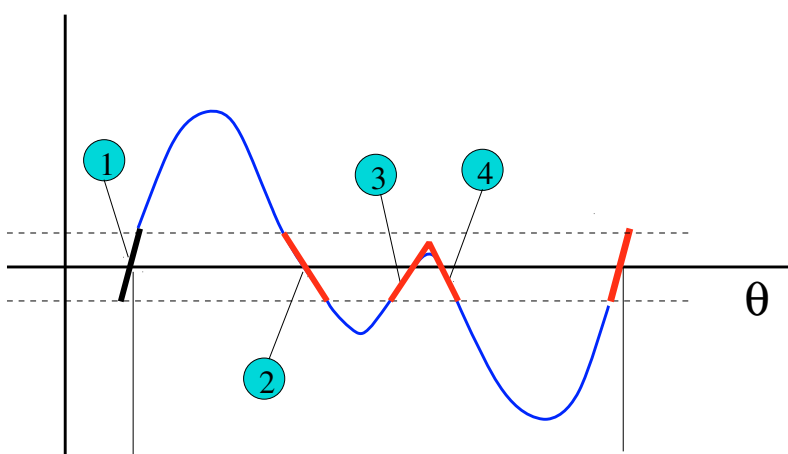

Fig. 2. The shape of $\frac{\mathrm{d} f_{0}}{\mathrm{~d} \theta}$ (blue line), and the local linear approximation at the image positions (red segments). The solution presented here is the simplest model consistent with the data. The numbers associated with each linear segments corresponds to the image numbers superimposed to the image of the lens (see Fig. 1).

the field $\frac{\mathrm{d} f_{0}}{\mathrm{~d} \theta}$ is more complicated and will be explained in details in this section. Note that the direct relation between the perturbative fields and the data is possible only for bright areas, and that as a consequence $\frac{\mathrm{d} f_{0}}{\mathrm{~d} \theta}$ and $f_{1}$ will have to be interpolated in dark areas. The derivation of the general properties of $\frac{d \tilde{f}_{0}}{\mathrm{~d} \theta}$ require a specific guess about the local behavior of this field in the region of image formation. Images form in a minimum of $\left|\frac{\mathrm{d} \tilde{f}_{0}}{\mathrm{~d} \theta}\right|$, the local behavior of $\frac{\mathrm{d} f_{0}}{\mathrm{~d} \theta}$ near the minimum is twofold: linear behavior for small images, for large images (caustics) $\frac{\mathrm{d} f_{0}}{\mathrm{~d} \theta}$ behaves like an higher order polynomial (see Alard 2009, for more details). As images of the source bright region are small (see Fig. 1), a local linear model will be adopted. The parameters of the local linear model are estimated with Eq. (5). Given the values of the four local slopes and the constraint that no images are formed in dark areas $\left(\frac{\mathrm{d} f_{0}}{\mathrm{~d} \theta}>\right.$ source radius), the general shape of the field is reconstructed (see Fig. 2). Note that the sign of the slope for two consecutive images has to be different to avoid a crossing of the zero line between the images and the formation of an additional image. Note also that at this level the sign of $\frac{\mathrm{d} f_{0}}{\mathrm{~d} \theta}$ is degenerate. Implicitly, since only one feature is seen in the upper right bright area, the current model assumes that the two bright spots visible in the other images are merged here. This is the simplest model which is consistent with the data. Another possibility is to assume that the two images are not merged and that the yellow contour in Fig. 1 corresponds to the image of the second bright spot, as considered by Tu et al. (2009). Still since the separation between the images is large the slope of the local model would have to be small. To be consistent with this small local slope and the presence of a dark area between the two images, a higher order Fourier expansion of $\frac{\mathrm{d} f_{0}}{\mathrm{~d} \theta}$ would be required (see Fig. 3). Additionally the color diagrams of the four bright spots (red contours in Fig. 1) are very similar, but the color diagram of the fifth bright spot (yellow contour in Fig. 1) is significantly different (see Fig. 4). This slight but significant difference in color shows that the fifth bright spot is not associated with the same source area as the other four. This is a confirmation that the solution proposed in Tu et al. (2009) is not consistent with the data, and that that the simplest perturbative model (Fig. 2) predicts the correct association of the images. 


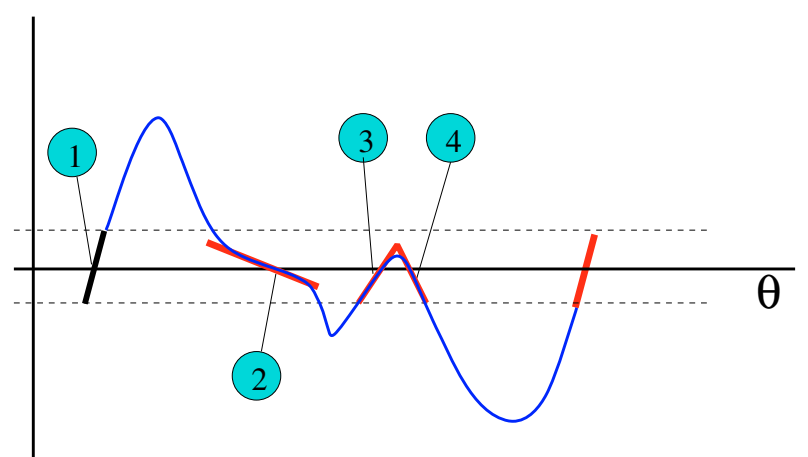

Fig. 3. The shape of $\frac{\mathrm{d} f_{0}}{\mathrm{~d} \theta}$ (blue line), and the local linear approximation at the image positions (red segments). The numbers associated with each linear segments corresponds to the image numbers superimposed to the image of the lens (see Fig. 1).

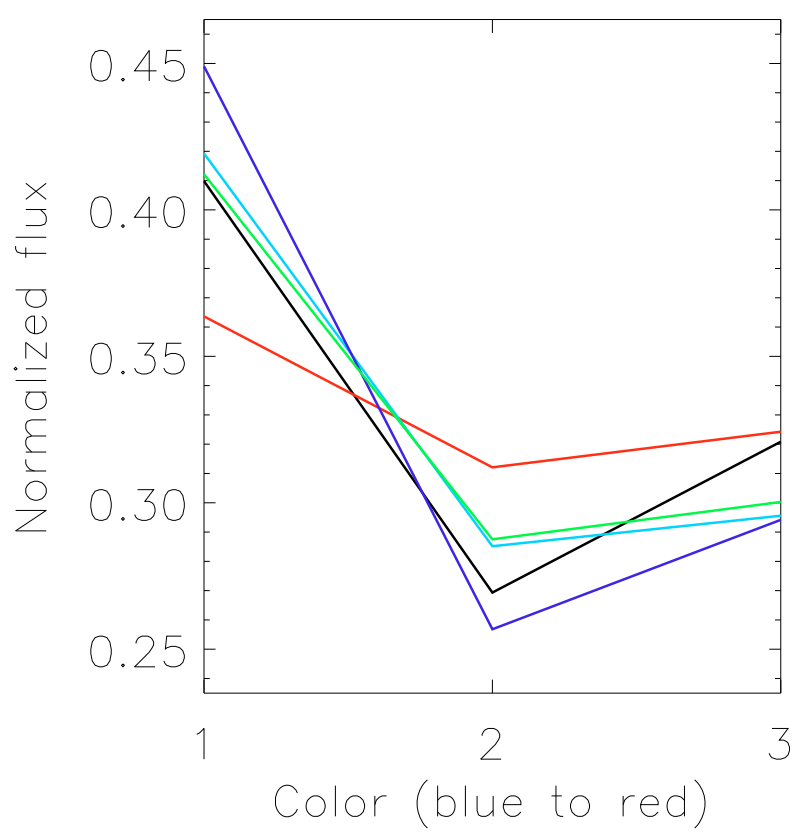

Fig. 4. The raw HST flux in each photometric bands normalized by the total flux in the three bands. The red line represents the color of the fifth bright area (yellow contour in Fig. 1), the other lines represent the color of the other four bright areas (red contours in Fig. 1). The color line of the fifth bright area is different at the $4 \sigma$ levels from the mean line of the other four bright areas.

\section{Fitting of the perturbative fields}

A qualitative evaluation of the perturbative fields has been performed in Sect. 4.3, and we will turn now to a quantitative evaluation of $\frac{\mathrm{d} f_{0}}{\mathrm{~d} \theta}$. The quantitative evaluation will be conducted in two steps, first an approximate guess will be made using the circular source solution, and then this guess will be refined by fitting the data.

\subsection{First numerical guess}

As explained in Sect. 4.3, the field $f_{1}$ in the circular source approximation is directly related to the mean image radial position. To reconstruct this field a Fourier series of order three is fitted to the mean image position. Fitting a higher order Fourier series does not significantly improve the fit, and a lower order expansion is not a good match to the data. Let us now turn to the

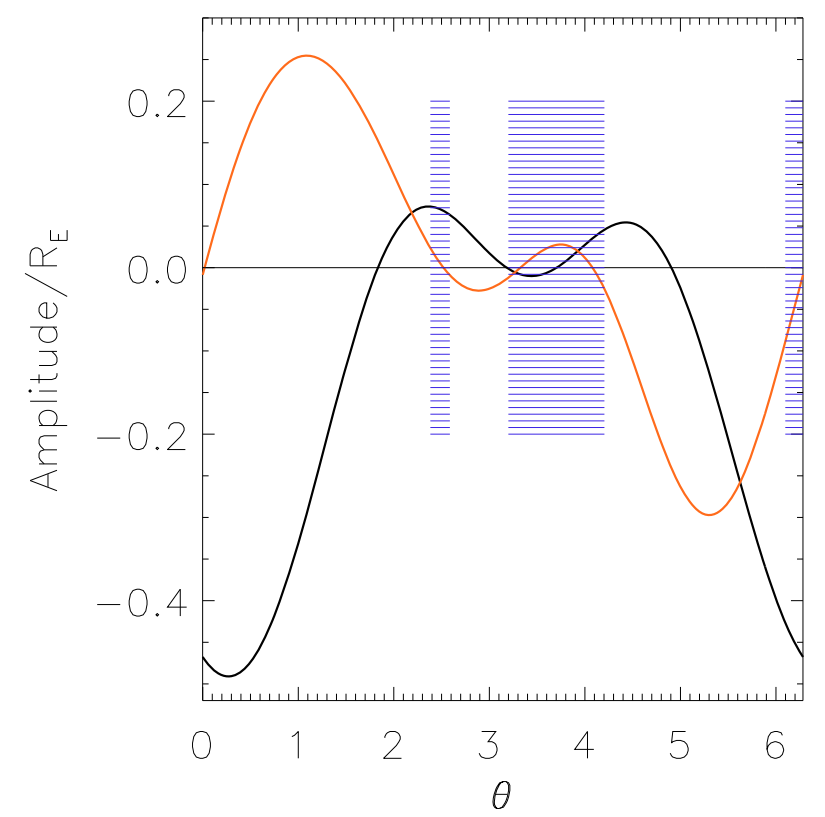

Fig. 5. The perturbative solution, $\frac{\mathrm{d} f_{0}}{\mathrm{~d} \theta}$ (red) and $f_{1}$ (black). The blue areas corresponds to the position of the images.

estimation of $\frac{\mathrm{d} f_{0}}{\mathrm{~d} \theta}$. The solution presented in the former section (see Fig. 1) is evaluated using the following piecewise numerical model: first order polynomials in each area where an image of the source is present, second order polynomials in dark areas. Equation (5) shows that the image angular size is given by the condition $\frac{\mathrm{d} f_{0}}{\mathrm{~d} \theta}=R_{0}$. Thus for a linear model, $k \frac{1}{2} \Delta \theta=R_{0}$, with $\Delta \theta$ the angular size of the image, and $k$ the slope of the linear model. The coefficients of the second order polynomials in the dark areas are evaluated using the continuity condition of the functions between the polynomial regions, the constraint that no images should be formed in dark areas $\left(\frac{\mathrm{d} f_{0}}{\mathrm{~d} \theta}>R_{0}\right)$, and the constraint that the solution should be as smooth as possible. The piecewise polynomial solution is fitted with an order three Fourier series. The Fourier expansion will be used as a first guess for the final fitting of the perturbative fields. The numerical guess are presented in Fig. 5. More details about the reconstruction of the numerical guess are available in Alard (2009), Sects. 4.3 and 4.4.

\subsection{Final fitting of the fields}

The final fitting of the fields is conducted by reconstructing the images of the source and comparing these images with the HST data by chi-square estimation. The reconstruction of the images for a given set of perturbative fields will be performed with the Warren \& Dye (2003) method. The source is represented with a linear combination of basis functions. The basis functions are identical to the functionals used for image subtraction (Alard \& Lupton 1998; Alard 2000). The images of each basis function are reconstructed and convolved with the HST PSF. The HST PSF is estimated with the Tiny Tim software (Krist 1995). Finally, the convolved images of the basis function are fitted to the HST data using a linear least-square method. The quality of the fit is evaluated by chi-square estimation. This fitting of the data for a given set of perturbative fields is conducted iteratively. The initial estimation starts from the numerical guess presented in the former section, leading to an initial chi-square estimation. This initial guess is improved iteratively using the Nelder \& Mead (1965) simplex method until convergence is reached. The final 


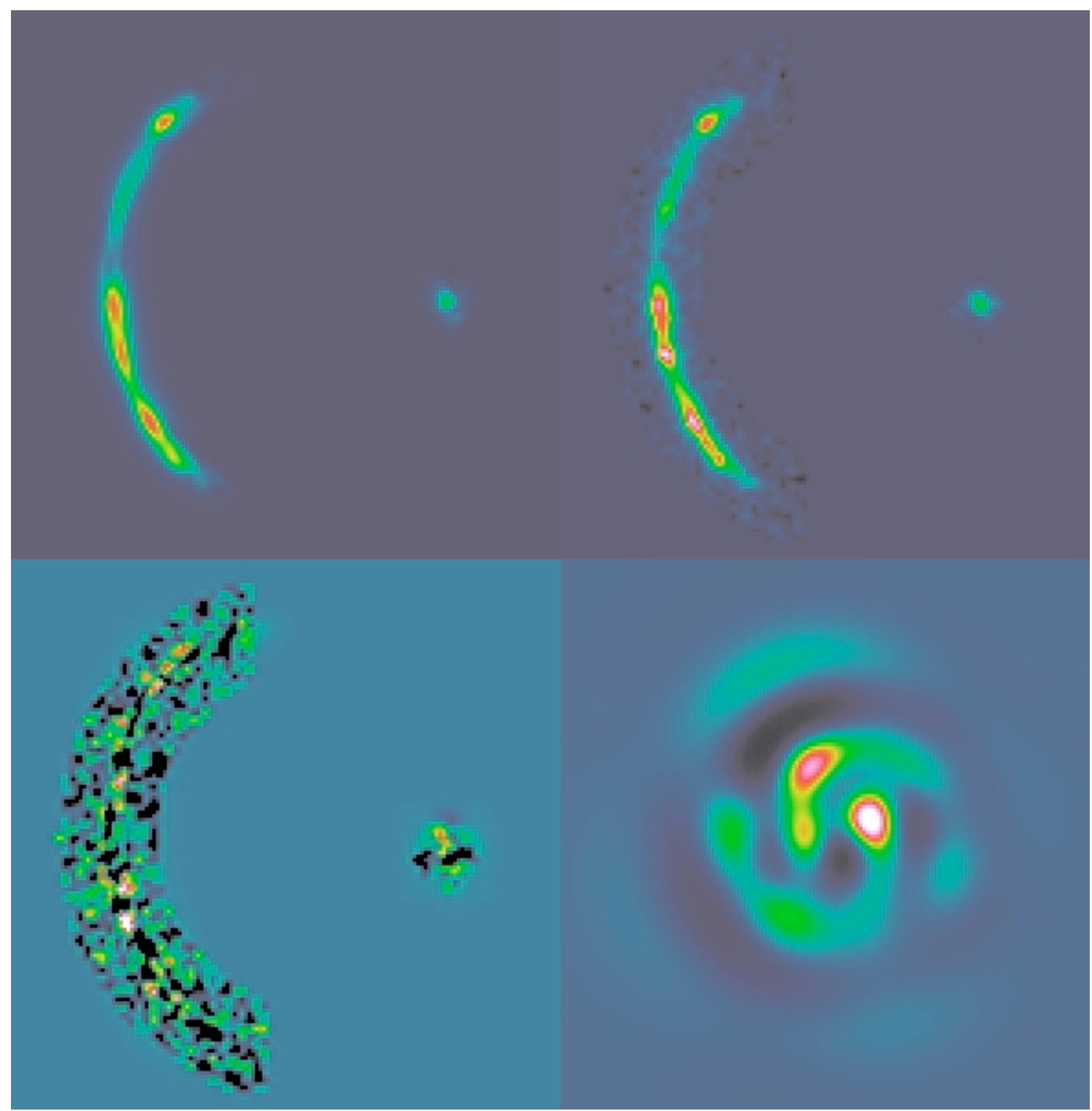

Fig. 6. Perturbative reconstruction of SL2S2176-0513. The HST image is on the upper right, the perturbative reconstruction on the upper left, the subtraction between the perturbative reconstruction and the HST data is on the lower left, the last image presents an image of the source.

result is presented in Fig. 6. The lens caustics are derived using Eq. (31) in Alard (2007), see Fig. 7. The source is situated near a cusp caustic with some parts of the source inside the caustic curve, and some part outside with different image multiplicity. A significant part of the source on the lower side of Fig. 7 is outside the caustic system and has a lower multiplicity. This part of the source is very close to the caustic, as a consequence one of its images is very elongated and corresponds to the image outlined by a yellow contour in Fig. 1. There is also a part of the source in the upper diagram, but this part is faint and more distant from the caustic, and the associated images are much smaller and fainter.

\subsection{Noise}

\subsubsection{Chi-square of the fit}

The model and the data are compared in a small region around the arcs with a total number of pixels $N$. The Poisson weighted difference $R_{i}$ between the model at pixel $i, M_{i}$ and the HST data $D_{i}$ is very close to a Gaussian (see Fig. 9). Considering that the model has $N_{\mathrm{P}}$ parameters, the 13 parameters of the perturbative expansion, and the 57 source model parameters, the chi-square is $\chi_{2}$ /d.o.f. $=\frac{1}{N-N_{\mathrm{P}}} \sum_{i} R_{i}^{2} \simeq 1.33$. Changing the size of the area by a small amount, either reducing it by moving closer to the center of the arcs, or enlarging the area, does not significantly change the chi-square value.

\subsubsection{Errors on the reconstruction of the perturbative fields}

Alard (2009) investigated the errors on the reconstruction of the perturbative fields and found that the errors due to the Poissonian noise were negligible. The reconstruction noise is dominated by the errors introduced by the first order approximation of the lens equation (see Alard 2009, Sect. 4.6). The results for SL2S021760513 are very similar, the mean error on the Fourier coefficients of the perturbative expansion due to the Poisson noise is $\sigma_{\mathrm{P}} \simeq 0.25 \times 10^{-3}$ (in units of the critical radius). The error due to the first order approximation $\sigma_{\mathrm{A}}$ is evaluated by comparing the position of the bright details in the HST image and the model reconstruction. The measurement procedure is described 


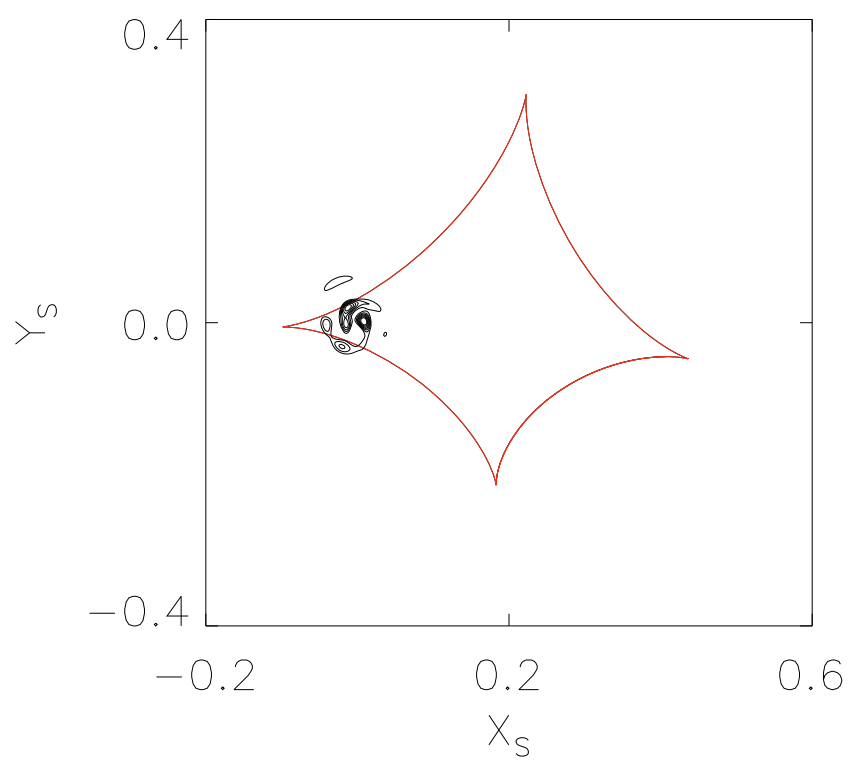

Fig. 7. The caustic system of the lens (red curve) and the source isocontours (black curve). The parts of the source inside and outside the caustics have different image multiplicity. Most of the images are associated with the two bright source regions situated inside the caustics. The fifth bright spot (yellow contour in Fig. 1) is associated with the lower part of the source situated below the caustic curve, very close to the curve. See Fig. 8 for a local zoom.

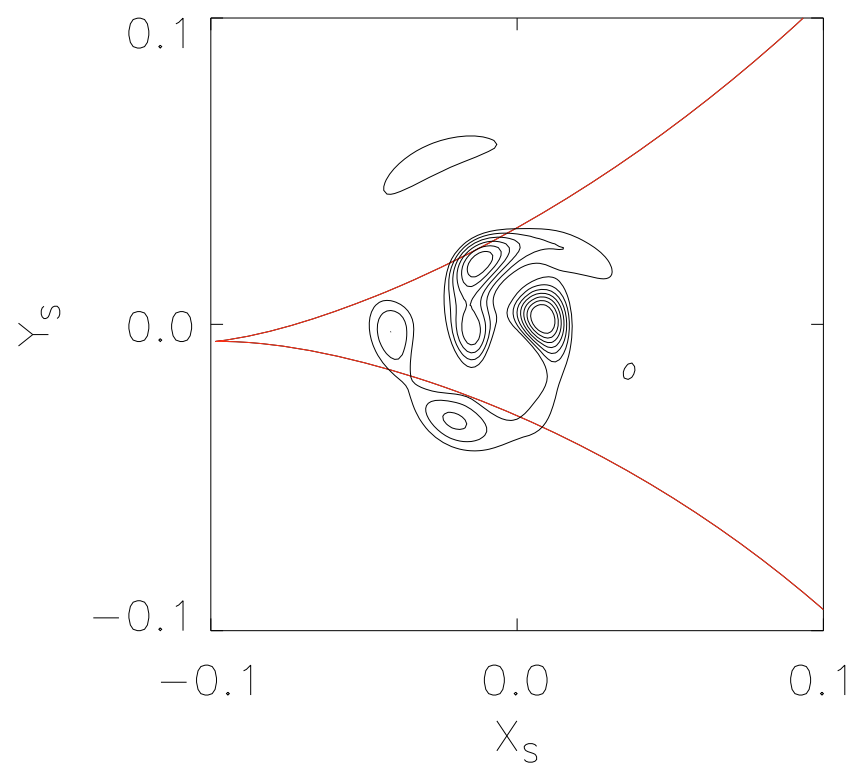

Fig. 8. A zoom image of the source and caustics.

in Alard (2009) Sect. 4.5.2. The numerical estimation of the error on the position between model and data is $\simeq 0.6 \% R_{\mathrm{C}}$, with $R_{\mathrm{C}}$ the critical radius. This positional error is identical to the error on the perturbative fields (see Alard 2009, Sect. 4.6.2). Assuming that the errors on the Fourier coefficient are similar, the error is $\sigma_{\mathrm{A}} \simeq 2.5 \times 10^{-3}$ which is much larger that the error due to the Poisson noise $\sigma_{\mathrm{P}}$. As a consequence the Poissonian noise will be neglected. Following Alard (2009) Sect. 4.6.3, the error on the reconstruction of the potential iso-contours $\delta \phi_{\text {iso }}$ is

$\delta \phi_{\text {iso }}=\sigma_{\mathrm{P}} \sqrt{\sum_{j=1}^{3} \frac{1}{j^{2}}} \simeq 0.0026$.

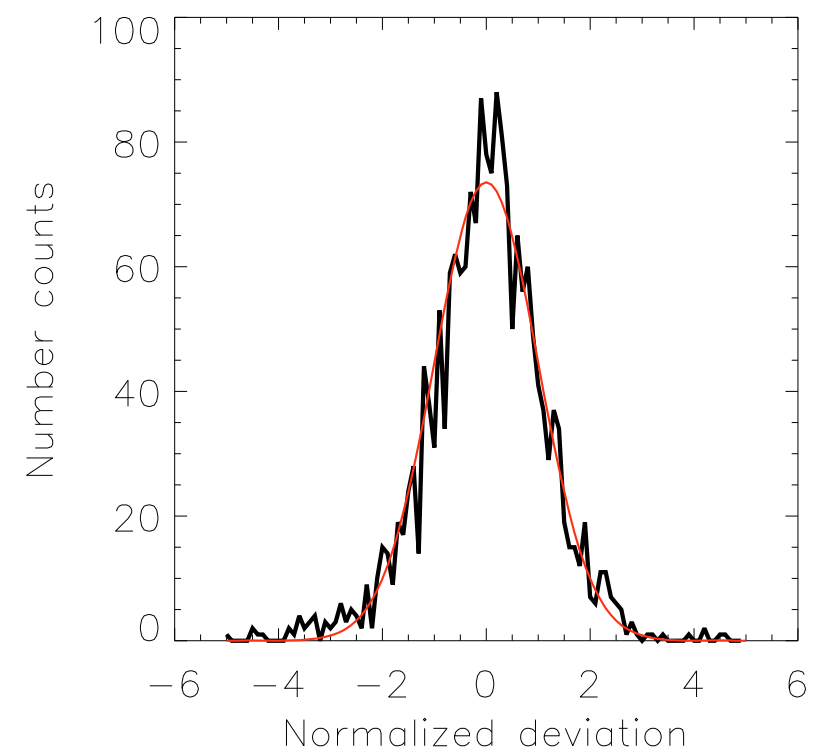

Fig. 9. Histogram of normalized residual (black curve) superposed to the Gaussian expectation (red curve).

\section{Properties of the lens}

The perturbative expansion is related directly to the properties of the lens potential. In particular Eq. (C.1) in Alard (2009) relates $f_{0}$ to the potential isocontours.

\subsection{Potential isocontours}

The solution of the perturbative lens equation (Eq. (5)) depends on $\tilde{f}_{i}=f_{i}+x_{0} \cos \theta+y_{0} \sin \theta, i=0,1$, and $x_{0}, y_{0}$, the source impact parameters. The potential isocontour is related to $f_{0}$ by the equation $\mathrm{d} r=-f_{0}$ (see Alard 2009, Eq. (C.1)). Since only $\tilde{f}_{0}$ is known, and since the impact parameters are unknown the first order Fourier terms of $f_{0}$ are unknown. The first order Fourier terms correspond to the centering of the potential and do not affect the shape of the potential isocontour (see Alard 2009, Sect. 6). As a consequence the shape of the potential isocontours will be computed with the Fourier expansion of $\tilde{f}_{0}$ from order two to the maximum order (order three). However, the inner potential isocontour does not depend on the impact parameters, as a consequence of which the relevant first order terms are meaningful. But the corresponding terms are very small, indicating that the center of the inner potential isocontour is very close to the center of light. An important asset of the singular perturbative approach is that the inner and outer contribution of the mass distribution to the potential can be separated. The potential generated by the lens projected density inside the critical circle corresponds to the coefficients $a_{n}$ and $b_{n}$ in the expansion of the potential (see Eqs. (B.1) and (B.2) in Alard 2009). The potential generated by the the projected density outside the critical circle corresponds to $c_{n}$ and $d_{n}$. Equation (B.3) in Alard (2009) relates the Fourier expansion of the perturbative fields to $a_{n}, b_{n}, c_{n}$ and $d_{n}$. As a consequence it is possible to reconstruct the potential corresponding to the projected density within and outside the critical circle. The potential near the critical circle is dominated by the outer contribution (see Fig. 10). Furthermore, the shape of the inner and outer potential are not the same. Both the ellipticity and orientation of the inner mass are very different. Using the second order Fourier terms it is straightforward to estimate the elliptical parameters for the inner and outer potential. The 


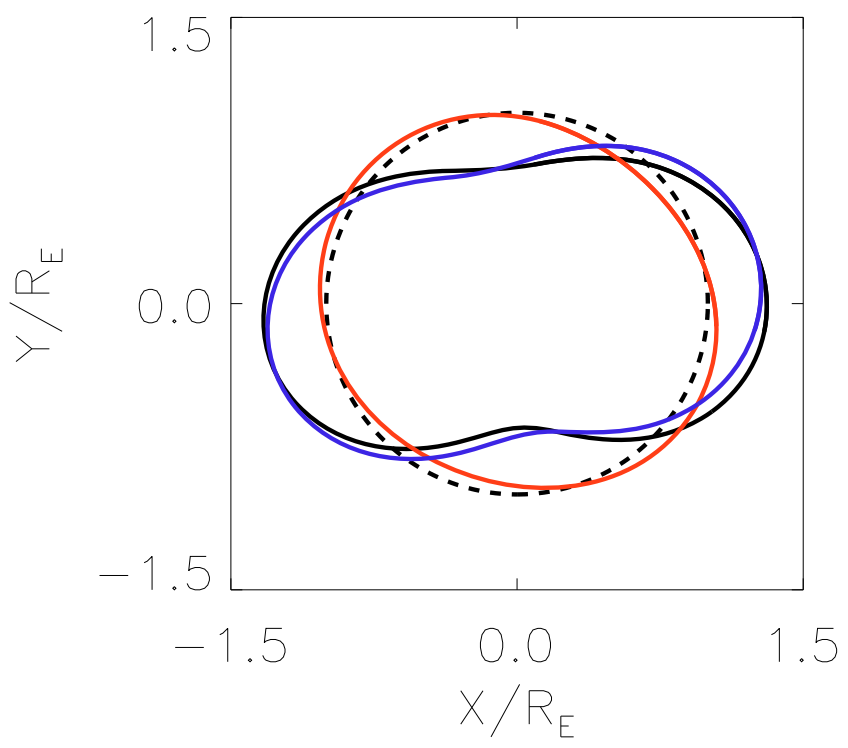

Fig. 10. The potential isocontours. The black curve corresponds to the potential generated by the whole mass distribution, the blue curve corresponds to the potential generated by the outer projected mass distribution and the red curve corresponds the potential generated by the projected mass distribution inside the critical circle. Note that the first order Fourier terms have not been included in the expansion (due to a degeneracy with the impact parameters). As a consequence the position of the contour center is unknown for the outer distribution. In this plot the direction of the vertical axis is parallel to the North (North is up and East is left). To improve the readability of the plot, the amplitude of the noncircular distortion has been exaggerated (by a factor of five).

elliptical contour equation in a coordinate system aligned with the ellipse axis reads

$(1-\eta) x^{2}+(1+\eta) y^{2}=R_{0}^{2}$.

To first order in $\eta$ :

$r=R_{0}\left(1+\frac{\eta}{2} \cos 2 \theta\right)$.

Considering that $r=1+\mathrm{d} r$, in a general coordinate system where the misalignment between the ellipse main axis and the abscissa axis is $\psi$

$\mathrm{d} r=\frac{\eta}{2} \cos (2(\theta-\psi))$.

An identification with the potential isocontour equation $\mathrm{d} r=-f_{0}$ leads to the inner mass contribution $\eta_{i}=0.028 \pm 0.005$ and $\psi_{i}=-31.7^{\circ} \pm 5^{\circ}$ and the outer contribution $\eta_{\mathrm{o}}=0.121 \pm 0.005$ and $\psi_{0}=9.79^{\circ} \pm 1^{\circ}$. Note that at first order in $\eta$ the ellipticity is equal to $\eta$. It is important to remind that as a consequence of the mass-sheet degeneracy the scaling factor $\kappa_{2}$ is unknown (see Eqs. (4) and (5)). In practice experiments with dark matter halos extracted form numerical simulations showed that $\kappa_{2} \simeq 1$ (Peirani et al. 2008). Note that $\kappa_{2}$ is a local quantity and that $\kappa_{2} \simeq 1$ does not require that the background potential is fully isothermal. It is recalled here that in this work $\kappa_{2}$ has been renormalized to unity for convenience (see Sect. 2). In practice $\kappa_{2}$ may be slightly different from unity, which would imply a re-scaling of the perturbative functionals. This rescaling would affect the ellipticity, but not other properties like the ellipse inclination angle.

\subsection{Comparison to observational properties of the lens}

It is interesting to compare the inner potential isocontour with the isocontours of the galaxy situated at the center of the lens. To estimate the photometric properties of the lens a Sersic profile was fitted to the light distribution. A similar procedure was already performed by Tu et al. (2009). The surface mass density profile is identical to Tu et al. (2009), Eq. (1), except that the quantity $\frac{b_{n}}{R_{e}^{1 / n}}$ is obviously degenerate and was replaced with a single parameter. The isocontour of this elliptical profile depends on $\eta$ (the ellipticity) and on the inclination angle of the ellipse main axis $\phi$. The fitting of the Sersic profile is conducted by minimizing the Poisson weighted residual between the Sersic model and a compilation of the three HST images. Note that the Sersic model is convolved with the HST PSF and that the nonlinear minimization is performed with Nelder and Mead (1965) simplex method. The results of this fitting procedure is $\eta=0.207 \pm 0.014$ and $\psi=-33.2^{\circ} \pm 2.4^{\circ}$. Tu et al. (2009) found: $\eta=0.14 \pm 0.06$ and $\psi=12 \pm 6^{\circ}$. The error bar was evaluated using a Monte-Carlo simulation (Poisson noise was added to the images). The ellipticity $\eta=0.207$ is compatible with Tu et al. (2009), but the consistency of the ellipse inclination is quite poor. The distance between the two measurements is larger than $3 \sigma$. Tu et al. (2009) suggest that the errors may be dominated by systematic effects. To investigate this issue the fit was performed separately for the three HST images. The results are (from blue to red photometric band): $\left(-35.1^{\circ},-44.9^{\circ}\right.$, $-25.1^{\circ}$ ), with an average of $35^{\circ}$ and a scatter of $9.9^{\circ}$. Note that estimating the scatter with such a small number of measurements is not very accurate. Considering the averaged value of the three measurements, no particular systematic is detected in this analysis. The reason for the different inclination angle found by $\mathrm{Tu}$ et al. (2009) is unclear. Considering the photometric properties found in the present work, the comparison with the iso-contours of the inner potential reads like this the difference in inclination is $1.5^{\circ} \pm 5.5^{\circ}$, the ellipticities are $0.207 \pm 0.014$ (galaxy) and $0.028 \pm 0.005$. Obviously the inclination angle is compatible, which suggests that the dark matter halo surrounding the galaxy has a similar orientation. The comparison of the ellipticities is model-dependent. The relation between the dark halo ellipticity and the ellipticity of the potential iso-contour is evaluated using the simplifying assumption that the density is dominated by an isothermal dark halo with ellipticity $\eta_{\mathrm{d}}$. By expanding the former density to first order in $\eta_{\mathrm{d}}$ we find that the ellipticity of the potential iso-contour is: $3 \eta_{\mathrm{d}}$. Thus if the ellipticity of the dark halo is the same as the ellipticity of the light distribution, the potential isocontour ellipticity would be $\eta=0.069 \pm 0.005$. The difference in ellipticity between the inner potential iso-contour is $0.04 \pm 0.007$. Thus the ellipticities are not consistent, and this simple model suggests that ellipticity of the dark halo is probably smaller than the ellipticity of the light distribution.

\subsubsection{Mass distribution outside the critical circle}

Let us now turn to the contribution of the outer projected distribution to the potential. Tu et al. (2009) pointed out that the central galaxy is close to a group of galaxies observed by Geach et al. (2007). The great difference between the outer and inner mass distribution is due to the influence of this group on the outer component. A simple model considers two isothermal components for the central galaxy dark matter halo and for the mass of the group. Let us assume that these two isothermal components 
have a velocity respectively dispersion of $\sigma_{0}$ and $\sigma_{1}$. The potential for an isothermal system then reads $\phi=c_{0} \sigma^{2} r$, where $c_{0}$ is a constant. The central galaxy is very close to the center of the coordinate system, thus

$\phi_{0}=c_{0} \sigma_{0}^{2} r$.

In a coordinate system where the center of the group is on the abscissa axis at a distance $r_{1} l l 1$, the potential of the group reads

$\phi_{1}=c_{0} \sigma_{1}^{2}\left|\boldsymbol{r}-\boldsymbol{r}_{1}\right|$

The critical condition implies that $\left[\frac{\partial \phi_{0}}{\partial r}\right]_{r=1}=1$, thus $c_{0}=\frac{1}{\sigma_{0}^{2}}$, and as a consequence

$\phi_{1}=\frac{\sigma_{1}^{2}}{\sigma_{0}^{2}}\left|\boldsymbol{r}-\boldsymbol{r}_{1}\right|$.

To estimate the contribution of the group to the field $f_{0}$, we will evaluate $f_{0}=\phi_{1}(r, \theta)$ at $r=1$. To second order in $\frac{r}{r_{1}}$

$\phi_{1}(r, \theta)=\frac{\sigma_{1}^{2}}{\sigma_{0}^{2}}\left(r_{1}+\frac{r^{2}}{4 r_{1}}-r \cos \theta-\frac{r^{2}}{4 r_{1}}\right)$.

The coefficient of the second order Fourier term of $f_{0}$ at $r=1$ is $-\frac{\sigma_{1}^{2}}{4 \sigma_{0}^{2} r_{1}}$. An identification with the isocontour equation (Eq. (6)) shows that

$\frac{\sigma_{1}}{\sigma_{0}}=\sqrt{2 \eta r_{1}}$.

In the former section it was estimated that $\eta=0.121$, and adopting the position of the center of the group proposed by Tu et al. (2009), $r_{1} \simeq 36$ (in units of the critical radius). As a consequence, $\frac{\sigma_{1}}{\sigma_{0}}=\simeq 2.95$. Geach et al. estimate that $\sigma_{1}=520 \pm$ $120 \mathrm{~km} \mathrm{~s}^{-1}$ from the $\sigma-T_{X}$ relation and $\sigma_{1}=770 \pm 170 \mathrm{~km} \mathrm{~s}^{-1}$ from the X-ray temperature. Tu et al. (2009) estimate from photometric data that $\sigma_{0}=218_{-28}^{+43}$. Assuming that the mass is dominated by the dark halo, this means respectively $\frac{\sigma_{1}}{\sigma_{0}}=2.38 \pm 0.72$ and $\frac{\sigma_{1}}{\sigma_{0}}=3.53 \pm 1.04$. Both value are compatible with $\frac{\sigma_{1}}{\sigma_{0}} \simeq 2.95$ inferred from the outer contribution to the potential estimated using the perturbative approach. Let us now estimate the compatibility between the inclination angles. The group center is situated in a direction at $\theta_{G}=16^{\circ}$ from the abscissa axis. Since the uncertainty of the group center position is $10 \operatorname{arcsecs}(\mathrm{Tu}$ et al. 2009) this translates into an error in $\theta_{G}$ of $10.3^{\circ}$. This angle should match the inclination angle of the elliptical contour of the outer potential which is $9.79^{\circ} \pm 1^{\circ}$. Considering the errors, these two angles are consistent.

\section{Discussion}

The reconstruction of the lens SL2S02176-0513 with the perturbative method indicates that the gravitational field in this lens is dominated by an outer contribution from a nearby group of galaxies. The separation of the inner and outer potential contribution using the perturbative method is free of any assumptions. Furthermore the perturbative analysis shows that the inner potential contribution is small but statistically significant. The orientation of the inner potential isocontour is consistent with the shape of the central galaxy luminosity contours, which illustrates the accuracy of the perturbative re-construction. The outer potential isocontour reconstructed with the perturbative method is consistent with the perturbation introduced by the group of galaxies. Both the ellipticity and orientation match the group properties. The analysis of this gravitational lens demonstrates the ability of the perturbative approach to separate the inner and outer mass contribution to the potential without making any particular assumptions. This lens is very special since the outer contribution to the potential is well identified and dominates other contributions to the outer potential. For other lenses the identification of the perturbators situated outside the critical radius is generally not obvious, and with conventional methods the ability to reconstruct the proper lens potential is compromised. Since the perturbative approach does not contain these flaws, and as the inner and outer contribution to the potential can be separated it is clear, that an unbiased reconstruction of the lens properties can be performed. This ability of the perturbative method to reconstruct the inner properties of the lens are especially useful to interpret complex gravitational lenses (see Alard 2009). This analysis will be also very useful in the statistical analysis of the perturbation introduced by substructures in dark matter halos (see Alard 2008; Peirani et al. 2008).

Acknowledgements. This work is based on HST data, credited to STScI and prepared for NASA under Contract NAS 5-26555.

\section{References}

Alard, C. 2000, A\&AS, 144, 363

Alard, C. 2007, MNRAS, 382, L58

Alard, C. 2008, MNRAS, 388, 375

Alard, C. 2009, A\&A, 506, 609

Alard, C., \& Lupton, R. 1998, ApJ, 503, 325

Bartelmann, M., \& Meneghetti, M. 2004, A\&A, 418, 413

Blandford, R. D., \& Kovner, I. 1988, Phys. Rev. A, 38, 4028

Clowe, D., Bradac, M., Gonzalez, A., et al. 2006, ApJ, 648, L109

Geach, J., Simpson, C., Rawlings, S., Read, A. M., \& Watson, M. 2007, MNRAS, 381, 1369

Krist, J. 1995, ASPC, 77, 349

Nelder, J., \& Mead, R. 1965, Comp. J., 7, 308

Saha, P., \& Williams, L. 2006, ApJ, 653, 936S

Tu, H., \& 9 co-hautors 2009, A\&A, 501, 475

Vegetti, S., \& Koopmans, L. 2009, MNRAS, 392, 945

Zhao, H. S., \& Qin, B. 2003, ApJ, 582, 2 